\title{
Satellite Image Enhancement Using Dual Tree Complex Wavelet Transform
}

\author{
C. Periyasamy \\ Department of Mathematics, AMET University, Chennai, India \\ Corresponding author, e-mail: eei@journal.uad.ac.id
}

\begin{abstract}
Drawback of losing high frequency components suffers the resolution enhancement. In this project, wavelet domain based image resolution enhancement technique using Dual Tree Complex Wavelet Transform (DT-CWT) is proposed for resolution enhancement of the satellite images. Input images are decomposed by using DT-CWT in this proposed enhancement technique. Inverse DT-CWT is used to generate a new resolution enhanced image from the interpolation of high-frequency sub band images and the input low-resolution image. Intermediate stage has been proposed for estimating the high frequency sub bands to achieve a sharper image. It has been tested on benchmark images from public database. Peak Signal-To-Noise Ratio (PSNR) and visual results show the dominance of the proposed technique over the predictable and state-of-art image resolution enhancement techniques.
\end{abstract}

Keywords: satellite image enhancement, DT-CWT, PSNR

\section{Introduction}

The application of satellite imagery is increases day by day due the development in the sensor technologies in weather forecasting, astronomy, geographical information. Satellite imaging is one of the challenging tasks for the researchers. The different satellite sensors are available in the very low resolution to high resolution range for data collection.

Gamma corrected adaptive knee transformation based on beta wavelet for satellite image enhancement is explained in [1]. Input image is decomposed into sub band images such as Low-Low (LL), Low-High (LH), High-Low (HL), and High-High (HH). Then input image with LL band coefficients are changed to get enhanced LL- band using adaptive knee transformation.

Advanced block based dwt technique for contrast enhancement of satellite images is presented in [2]. Flexible radiant transfiguration and preeminent glaze levels are used in this method. Inverse Discrete Wavelet Transform (IDWT) is used to enhance the image finally and DWT based on advanced block is used to improve each block. Inverse DWT is used to fuse the enhanced blocks to obtain emanated image. Picture is decayed into sub bands using haar wavelet transform.

Fusion and Morphological Gradient based on DWT-Principal Component Analysis (PCA) for edge Preserving Satellite Image Enhancement is described in [3]. Input image is decomposed into various sub bands using DWT. Fusion is applied on the LL sub band using PCA. Then enhanced image is reconstructed using IDWT. Fine detail sub bands are required to achieve sharp boundary. Satellite image enhancement using an effective method is discussed in [4]. DWT with high-frequency sub bands and low resolution input image is used to obtain sharp image by high frequency sub band estimation. Resultant image is reconstructed using IDWT. Singular Value Decomposition (SVD) and DWT based on Gamma Correction for satellite image enhancement are explained in [5]. Intensity transformation based low contrast satellite images are enhanced. There are four various sub bands are included while decomposes the input image i.e. $\mathrm{LL}, \mathrm{LH}, \mathrm{HL}$ and $\mathrm{HH}$. Edge information is preserved by applying gamma correction. LL sub-band information of gamma is passed via SVD and IDWT is applied to reconstruct the enhanced image.

Plateau histogram equalization based satellite image contrast enhancement algorithm is presented in [6]. Input image decomposition is done using bi-histogram equalization with plateau and threshold calculation using self-adaptive plateau histogram equalization. Minimum 
mean brightness error bi-histogram equalization, histogram equalization, dynamic histogram equalization, self-adaptive plateau histogram equalization are compared with existing methods.

In this paper also described in, Land use and land cover classification of LISS-III satellite image using KNN and decision tree [7]. Combine technique for classification of IRS P6 LISS-III satellite images [8]. Pre-processing technique for the wireless capsule endoscopy image enhancement is discussed in [9]. Speech enhancement based on wiener filter and compressive sensing is discussed in [10]. IR and multi scale retinex image enhancement for concealed weapon detection is discussed in [11].

In the enhancement of the satellite image there may be some problems occurred during the transmission of image from one place to the other. Due to this there may be information loss in the images. So to avoid this we have proposed a solution in order to do the enhancement without any difficulty.

\section{Proposed Solution}

Low-pass filtered signal having some high frequency information because the analysis filter bank has finite filter taps and also some low frequency information is obtained from high pass filtered signal. Same phase has down sampling the both high-pass and low-pass filtered signals but still remains some correlation though, there will be correlation at low while down sampling by various phases. Image enhancement is done using DT-CWT technique to obtain a resolution-enhanced image. Results show that the proposed technique performs better than the existing wavelet methods in terms of the PSNR.

Figure 1 shows the block diagram of the proposed satellite image resolution enhancement system. An input image is decomposed by DT-CWT to get high-frequency sub bands. The high-frequency sub bands and the low-resolution input image are interpolated. Two different DWT decompositions are used to calculate the complex transform using DTCWT.

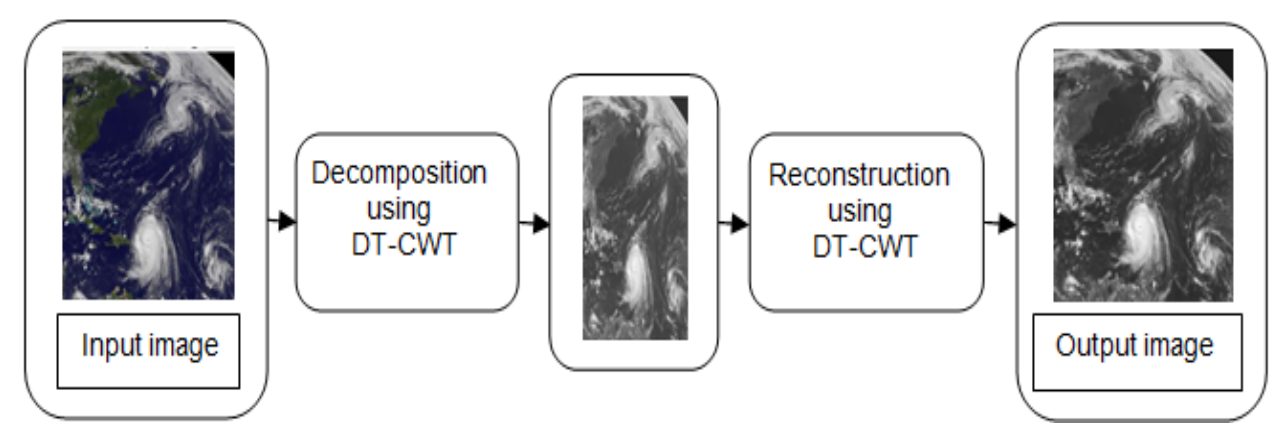

Figure 1. Block diagram of the proposed system

\section{Results and Discussion}

Number of well-known satellite test images is experimented. This section tells about the experimental results of the proposed contrast enhancement for satellite images. PSNR is calculated using satellite image. Satellite input image and output images are shown in Figure 2. Our results show that the proposed technique provides reliable improvements. Proposed PSNR value is $31.98 \mathrm{db}$. 


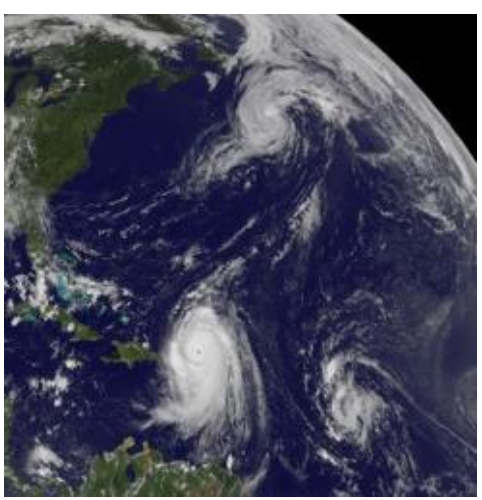

(a)

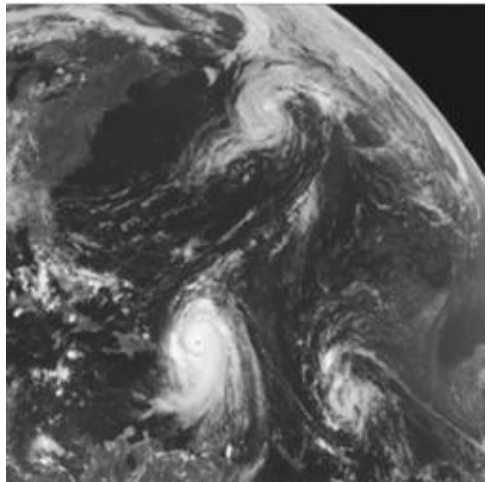

(b)

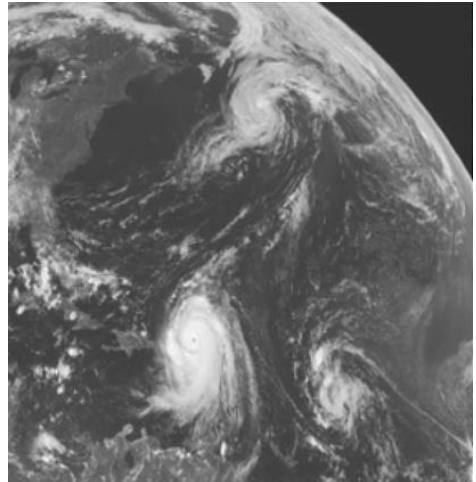

(c)

Figure 2. (a) Input image, (b) Enhanced image and (c) Reconstructed image

\section{Conclusions}

DT-CWT domain based image resolution enhancement algorithm is presented. Input images are decomposed using this proposed technique. Then decomposed images are enhanced and finally reconstructed using IDT-CWT. Our results have shown that the proposed method outperforms conservative image enhancement approaches. Figure 2 shows the proposed input and resultant images.

\section{References}

[1] Singh H, Kumar A. Satellite Image Enhancement Using Beta Wavelet Based Gamma Corrected Adaptive Knee Transformation. IEEE International Conference on Communication and Signal Processing. 2016; 0128-0132.

[2] Coumar SO, Rosario VV. Contrast Enhancement of Satellite Images Using Advanced Block Based DWT Technique. International Journal of Engineering and Future Technology. 2016; 3(3): 1-10.

[3] Thriveni R. Edge Preserving Satellite Image Enhancement Using DWT-PCA Based Fusion and Morphological Gradient. IEEE International Conference on Electrical, Computer and Communication Technologies. 2015; 1-5.

[4] Jadhav BD, Patil PM. An Effective Method for Satellite Image Enhancement. IEEE International Conference on Computing, Communication \& Automation. 2015; 1171-1175.

[5] Sharma N, Verma OP. Gamma Correction Based Satellite Image Enhancement Using Singular Value Decomposition and Discrete Wavelet Transform. IEEE International Conference on Advanced Communication Control and Computing Technologies. 2014; 1286-1289.

[6] Aedla R. Satellite Image Contrast Enhancement Algorithm Based on Plateau Histogram Equalization. IEEE Region 10 Symposium. 2014: 213-218.

[7] Upadhyay A, Shetty A, Singh SK, Siddiqui Z. Land Use and Land Cover Classification of LISS-III Satellite Image Using KNN and Decision Tree. IEEE 3rd International Conference on Computing for Sustainable Global Development. 2016:1277-1280.

[8] Upadhyay A, Singh SK, Kambli S, Combine Technique for Classification of IRS P6 LISS-III Satellite Images. International Journal of Control Theory and Applications. 2015; 9(10): 4293-4299.

[9] Shahril R, Baharun S, Islam AM. Pre-processing Technique for Wireless Capsule Endoscopy Image Enhancement. International Journal of Electrical and Computer Engineering (IJECE). 2016; 6(4): 1617-1626.

[10] Sulong A, Gunawan TS, Khalifa OO, Kartiwi M, Ambikairajah E. Speech Enhancement based on Wiener Filter and Compressive Sensing. Indonesian Journal of Electrical Engineering and Computer Science (IJEECS). 2016; 2(2): 367-379.

[11] Hussein NJ, Hu F, Hu H, Rahem AT. IR and Multi Scale Retinex image Enhancement for Concealed Weapon Detection. Indonesian Journal of Electrical Engineering and Computer Science (IJEECS). 2016; 1(2): 399-405. 